$\overbrace{\substack{\text { songes } \\ \text { colose }}}^{\infty}$

\title{
Current state of emergency medicine education in China
}

\author{
J F Shao, H Y Shen, X Y Shi
}

Second Affiliated Hospital of Zhejiang University College of Medicine, Hangzhou, Zhejiang Province, China

Correspondence to: Miss H Y Shen, Second Affiliated Hospital of Zhejiang University College of Medicine 88 Jiefang Road, Hangzhou, Zhejiang Province, China 310009; nancysjf@163.com

Accepted 16 November 2008

\section{ABSTRACT}

In China the practice of emergency medicine includes patient management in the prehospital, emergency department and intensive care settings. In recent years, emergency medicine has emerged as an independent medical specialty in its own right, and has built up its own professional pool of clinicians, academicians and researchers. There is, however, still much room for improvement compared with developed countries, especially in the areas of clinical and prehospital care, teaching and scientific research. In this paper the current state of emergency medicine education in China is presented and further avenues for improvement are explored.

The Ministry of Health and the Ministry of Education in China officially recognised emergency medicine as an independent medical discipline in 1983 and legislated establishments of independent emergency departments within all acute care hospitals. ${ }^{1}$ The development of emergency medicine has accelerated in recent years, and many departments have developed capabilities to handle prehospital, emergency department and intensive care unit patients. At the same time, a professional pool of clinical, research and teaching specialists has been built up.

Compared with developed countries, however, ${ }^{2}$ there remains much room for improvement in emergency medical team building, scientific research, teaching or clinical and prehospital emergency management.

At present, many emergency departments in China are staffed by doctors from other disciplines, and levels of training are uneven. Emergency medicine education is therefore paramount to ensure appropriate standards, and this includes on-the-job training (OJT) as well as continuing medical education.

\section{EMERGENCY MEDICINE PRACTICE AND EMERGENCY MEDICINE SERVICE SYSTEM (EMSS) IN CHINA}

There are three different types of emergency department in China. In the first model (the independent model), emergency physicians are fully responsible for assessment, treatment, observation, discharge and admission of patients in the emergency department. In the second model (the semi-independent model), the emergency department is staffed by a mixture of emergency physicians as well as doctors rotated from other disciplines. In this model the emergency physician is mainly in charge of observation and transfer of patients to appropriate departments. In the third model there are no trained emergency physicians and patients are seen directly by doctors from the relevant disciplines. At present, emergency departments in most hospitals are of the semi-independent model.

Staff working in the prehospital or Emergency Medicine Service System (EMSS) include medical doctors, registered nurses, patient carriers and drivers. ${ }^{3}$ At present there are four EMSS models: Beijing, Shanghai, Chongqing and Guangzhou. In the Beijing model the emergency department, ambulance stations and dispatch communication centre are linked; patients are transferred to emergency departments or hospitals within the same network. Beijing Emergency Medical Center (Beijing EMC) is responsible for the prehospital treatment of the whole of Beijing. Beijing EMC boasts a modern medical aid command centre, 75 first-aid stations, 10 suburban first-aid substations, hundreds of ambulances and a group of professional medical staff, which collectively constitute the medical aid system outside the hospital, along with 110 alarms, 119 fire alarms, 120 ambulances, public transportation, subway and airport and so on, to build an interactive system for ensuring the security and stability of Beijing. The Shanghai model is primarily a prehospital emergency system where dispatch centres coordinate transfer of patients to the nearest hospitals. In the Chongqing model the whole hospital serves as an emergency department which is divided into many departments (eg, internal medicine department, surgery department, intensive care unit). The ambulance staff are employed and managed by the hospital. Hospital staff provide prehospital care. In the Guangzhou model, ambulances are hospital-based; a communication centre coordinates dispatch of vehicles from the nearest hospital for patient rescue.

\section{CURRENT STATE OF EMERGENCY MEDICINE EDUCATION IN CHINA}

To date, the training of emergency department doctors in most parts of China has not been formalised. Doctors who join emergency departments are usually new graduates from other disciplines or have been transferred from other hospitals. They may then be rotated to other disciplines for further training for a period of $1-$ 3 years (the training period is decided by the department and not the physicians themselves). An examination is usually held at the end of the training period.

The first national emergency care residency programme in China was set up at the Peking Union Medical College Hospital in 1986. Currently 5-10 graduate medical doctors are enrolled each 
year. On completion of the 3-year programme, qualified emergency physicians are assigned to work in various emergency departments throughout the country. Each province has its own residency training programme.

\section{Emergency medicine education curriculum}

At present, emergency medicine education is carried out via three main channels: (1) undergraduate education; (2) postgraduate education; and (3) continuing medical education (CME) for existing specialists.

\section{Undergraduate education}

Compulsory or elective courses on emergency medicine are included in the curriculum for undergraduate medical students. The contents of the course include life support and emergency management for acute medical, surgical and paediatric conditions. One of the earliest examples of the textbook used for this course was "Emergency Medicine" edited by the Chinese Medical Association in 1989. The students may also undergo internationally recognised courses such as Advanced Cardiac Life Support (ACLS) and Advanced Trauma Life Support (ATLS).

\section{Postgraduate education}

Many colleges now offer Masters Degrees in emergency medicine for those training to be emergency medicine specialists. In August 2003, a programme for a Doctor's Degree in Emergency Medicine started at the Second Affiliated Hospital of Zhejiang University. This is the first doctorate programme for emergency medicine in China. However, the number of doctorate degree holders is still low. For example, at the emergency centre of the Second Affiliated Hospital of Zhejiang University (currently the biggest emergency centre in China), only 7 out of 25 emergency physicians (28\%) have a Doctor's Degree in Emergency Medicine.

\section{Continuing medical education (CME) for existing specialists}

Emergency physicians receive continuing medical education via OJT, emergency medicine conferences and expert lectures on emergency medicine practices. Senior emergency physicians also attend courses to improve their teaching, research and management skills.

These different forms of emergency medicine education for different levels of trained specialists at various stages of their career provide the emergency physicians with opportunities for further development and keep them up to date with the latest practices. $^{4}$

As a sideline, emergency physicians do not use traditional Chinese medicine (TCM) in their practices, even those working at TCM institutions. This is mainly because TCM has a slow onset of action and is usually not useful in an acute setting.

\section{Emergency medicine education in Zhejiang Province}

Pioneering work to establish emergency medicine education systems, training objectives, curriculum and teaching materials was carried out all over China in the 1980s when formal emergency medicine education was first introduced.

The first emergency medicine education project in Zhejiang Province started in 1984 when Health Opportunities for People Everywhere (Project HOPE) funded two emergency medicine nurses from the Second Affiliated Hospital of Zhejiang University College of Medicine to go to the USA for a 6-month period of training in emergency medicine. In 1989 the
Emergency Medicine Guidance Center was established in Zhejiang Province with the support of Project HOPE. HOPE provided the centre with advanced equipment and arrangements were made for an American-trained clinician to work at the centre. An OJT programme for emergency physicians was also started. Doctors who completed the programme were awarded emergency training certification. In the same year the first elective course in emergency medicine for undergraduate medical students was started at the Zhejiang University College of Medicine. In 1998 the national CME point system for emergency medicine training was established by the joint efforts of the Emergency Medicine Society of Zhejiang Medical Association and Zhejiang University College of Medicine. Doctors participating in emergency medicine training were accredited CME points; these points were important to the physicians' prospects for promotion.

Currently there are four levels of emergency medicine education for doctors in Zhejiang Province. These include an introductory course in emergency medicine during undergraduate education, CME programmes for practising doctors and Masters and Doctorate programmes for emergency medicine specialists.

\section{CME programmes in Zhejiang Province}

$\mathrm{CME}$ is an integral part of emergency medicine education. As emergency medicine is still a young medical specialty in China, most specialists tend to be in the younger and middle-aged groups. Most physicians, including the most senior staff, actively participate in CME programmes to keep up with the latest developments and to upgrade their skills. In Zhejiang Province the mainstay of CME programmes includes OJT, academic conferences and correspondence courses.

We compared the attendees at three CME programmes in Zhejiang Province: (1) OJT sponsored by the Emergency Medicine Guidance Center of Zhejiang Province in 2005; (2) correspondence course sponsored by the Chinese Journal of Emergency Medicine in 2005; and (3) the Academic Conference on Emergency Medicine sponsored by the Chinese Journal of Emergency Medicine in 2006.

A total of 892 persons were registered in the three projects, and information on 798 was complete. Using $\chi^{2}$ analysis, we found significant differences in the age of the attendees for these programmes $(p<0.01)$ with participants in the correspondence course being younger (25-34 years) than those participating in OJT and the academic conference (35-44 years). We postulated that it was easier for senior doctors to get time off to attend conferences, while younger doctors need to study in their own time.

\section{COMPARISON OF EMERGENCY MEDICINE EDUCATION IN CHINA WITH OTHER DEVELOPED NATIONS}

In developed nations such as the USA, knowledge of EMSS is emphasised during the emergency medicine residency programme..$^{5}$ Unfortunately, training in EMSS is not currently well established in China.

In the USA the various emergency medicine boards (eg, American Board of Emergency Medicine (ABEM), American College of Emergency Physicians (ACEP), Council of Emergency Medicine Residency Directors (CORD), Emergency Medicine Residents' Association (EMRA), The Residency Review Committee of Emergency Medicine (RRC-EM) and the Society for Academic Emergency Medicine (SAEM)) issue policy statements guiding emergency medicine clinical practice and education. This is currently lacking in China. 


\section{AVENUES FOR IMPROVING EMERGENCY MEDICINE EDUCATION Diversity in training}

Current emergency medicine education in China is varied and diversified. Many education programmes-including full-time and part-time courses, correspondence courses and remote network education - are run by various medical colleges, medical associations and medical media. Besides clinical and academic courses, there are courses catering for the development of research, applications and technical skills. Training in a number of aspects of emergency medicine allows physicians to develop their own areas of interest and expertise, and helps emergency medicine to advance as a medical specialty on the whole.

\section{CME: the core of emergency medicine education}

China is still a developing nation and there is an imbalance in the levels of medical training in different areas of the country. For most regions of China the greatest barrier to development of emergency medicine is the lack of funding. CME programmes help to overcome part of this barrier and lay down the infrastructure for the advancement of emergency medicine. CME is thus the core of emergency medicine education for China in the 21st century.

Through OJT training programmes, OJT junior staff are taught the basics of resuscitation and acute management of emergency conditions. Improving these skills in junior staff is especially important in view of the fact that sudden death due to cardiovascular and cerebrovascular diseases and trauma has increased in China over recent years.

By formulating and conducting CME programmes, emergency medicine practitioners are able to build up expertise in training and teaching and further develop academic emergency medicine.

\section{Developing an infrastructure and quality control for CME}

With the increased complexity and sophistication of CME, demands for specialised teaching and quality control have increased. There is an urgent need to accelerate the development of an infrastructure for $\mathrm{CME}$ in emergency medicine. We propose a three-pronged approach:

- Medical colleges, emergency medicine institutions, emergency medicine societies and academic bodies should build on current $\mathrm{CME}$ resources to improve the quality of teaching.

- These different bodies should interact and work together, especially in areas where programmes overlap. Academicians with expertise in the subject should be asked to help in improving and updating the CME content.

- Other relevant specialties should be involved in the development of academic emergency medicine.

Funding: This paper was supported by grant number 5XW02 on the publishing industry cluster and economic and social development of the Yangtze River delta.

Competing interests: None.

\section{REFERENCES}

1. Chen $\mathbf{X Y}$. Pay attention to standardized training of emergency medicine residents. Int J Emerg Crit Care Med 2007;4:2187.

2. Qiu $\mathbf{0 M}$, Lu ZO, Huang WJ, et al. The discussion on training of highly qualified emergency medicine doctors. J Clin Emerg Call 2006;7:201-3.

3. Zhao W. The emergency rescue role of emergency medical service system in disasters. Chin J Crit Care Med 2003;23:314-5.

4. Houry DE, Pons PT. The value of the out-of-hospital experience for emergency medicine residents. Ann Emerg Med 2000;36:391-3.

5. Hockberger RS, Binder LS, Graber MA, et al. The model of the clinical practice of emergency medicine. Ann Emerg Med 2001;37:745-70. 